\title{
ER-positive breast cancer patients with more than three positive nodes or grade 3 tumors are at high risk of late recurrence after 5-year adjuvant endocrine therapy
}

\author{
This article was published in the following Dove Press journal: \\ OncoTargets and Therapy \\ 3 October 2017 \\ Number of times this article has been viewed
}

\section{Fangbin Song' \\ Jianbing Zhang ${ }^{2}$ \\ Shanbao $\mathrm{Li}^{\prime}$ \\ Junyi Wu' \\ Tao Jin' \\ Jun Qin' \\ Ye Wang' \\ Min Wang' \\ Junming $\mathrm{Xu}^{\prime}$}

'Department of General Surgery, ${ }^{2}$ Department of Pathology, Shanghai General Hospital, Shanghai Jiao Tong University School of Medicine, Shanghai, People's Republic of China

Correspondence: Junming $\mathrm{Xu}$

Department of General Surgery, Shanghai General Hospital, Shanghai Jiao Tong University School of Medicine, No 100 Haining Road, 200080, Shanghai, People's Republic of China Email xujmsh@hotmail.com
Purpose: Currently, although several clinical trials available give strong suggestions that extension of endocrine therapy has benefits, the risk level at which patients may benefit from extended endocrine therapy remains uncertain. This study aimed to identify the proportion of patients at a substantial risk of late recurrence after 5-year adjuvant endocrine therapy.

Patients and methods: We reviewed 1,056 female patients with primary breast cancer who underwent curative resection between January 2006 and December 2011. Univariate and multivariate analyses were performed using the Cox proportional hazards regression model to identify prognostic factors.

Results: A total of 327 eligible patients were eventually enrolled in this study. Among them, 42 (12.8\%) patients suffered from distant metastasis and 34 (10.4\%) patients experienced locoregional recurrence after 5-year adjuvant endocrine therapy. In multivariate analysis, patients with more than three positive nodes (hazard ratio $[\mathrm{HR}]=2.176,95 \% \mathrm{CI}=1.071-4.421 ; P=0.032$ ) and histologic grade 3 disease $(\mathrm{HR}=2.098,95 \% \mathrm{CI}=1.300-3.385 ; P=0.002)$ were significantly associated with high risk of late recurrence. However, only histologic grade 3 (HR=2.212, 95\% $\mathrm{CI}=1.166-4.194 ; P=0.015$ ) was significantly associated with high risk of distant metastasis. Conclusion: Late relapse after completion of 5-year adjuvant endocrine therapy was still common, and grade 3 and more than three positive nodes were the risk factors of late recurrence, while grade 3 was the only risk factor of late distant metastasis. These patients might benefit from extended endocrine therapy.

Keywords: Ki-67, breast neoplasms, prognosis, extended endocrine therapy, tamoxifen, aromatase inhibitors

\section{Introduction}

Endocrine therapy is one of the mainstream treatments of breast cancer, and 5 years of adjuvant tamoxifen has long been considered the standard treatment for early-stage estrogen receptor (ER)-positive breast cancer and significantly reduces ER-positive breast cancer recurrence and mortality throughout the first 10 and 15 years, respectively. ${ }^{1,2}$ However, the optimal duration and treatment strategies involving adjuvant endocrine therapy in early breast cancer remain controversial. ${ }^{3,4}$ ER-positive tumors initially have low recurrence rates and a constant and unrelenting risk of relapse that extends up to 15 years. ${ }^{1,5}$ Several clinical trials have emerged to support extended endocrine therapy, as it becomes clear that the recurrence risk of breast cancer does not decrease beyond the initial 5 years of treatment. ${ }^{2,4,6-9}$ 
Fisher et al showed that continuous adjuvant tamoxifen treatment for 10 years vs 5 years led to adverse outcomes, and that an extension of endocrine therapy for an additional 5 years is likely to lead to an increase in side effects. ${ }^{6}$ Generally, tamoxifen is well tolerated, with the most reported events being hot flushes, osteoporosis, arthralgia and gynecologic symptoms such as vaginal bleeding and discharge. ${ }^{7}$ More severe toxicities are venous thromboembolisms, endometrial carcinomas and mood change or depression. ${ }^{10,11}$ For aromatase inhibitors (AIs), hypertension, dyslipidemia, arthralgia and osteoporosis are more frequently described and gynecologic symptoms and hot flushes are less common. ${ }^{7,12}$ Thus, the additional benefits of extending the endocrine therapy need to be appropriately balanced against the risk of side effects.

Furthermore, ER-positive breast cancer is a biologically heterogeneous disease as different biologic types may respond differently to the endocrine treatment. ${ }^{13,14}$ Clinical management of patients should be individualized, with the patient being a key factor in decision making. Currently, the subpopulation of patients who will actually benefit from extended therapy remains unclear, leaving clinicians and patients with uncertainty about whether to continue endocrine therapy. Consequently, we conducted this study to identify the proportion of patients at a substantial risk of late recurrence after 5-year adjuvant endocrine therapy, who might benefit from extended endocrine therapy.

\section{Patients and methods}

\section{Patient selection}

A total of 1,056 female patients with primary breast cancer who underwent curative resection at the Shanghai General Hospital (Shanghai, People's Republic of China) between January 2006 and December 2011 were reviewed. The inclusion criteria were as follows: 1) positive ER immunohistochemical (IHC) results; 2) stage I-III infiltrating breast carcinoma and 3) completion of 5-year adjuvant endocrine therapy (tamoxifen or AIs). The exclusion criteria were: 1) no endocrine therapy or endocrine therapy $<5$ years; 2) metastasis or recurrence, died or lost to follow-up before completion of 5-year endocrine therapy; 3 ) endocrine therapy $>5$ years; 4 ) neoadjuvant therapies before primary surgery and 5) tamoxifen switch to AIs or AIs switch to tamoxifen. Eventually, 327 eligible patients were enrolled in this study (Figure 1). The study was approved by the Ethics Committee of Shanghai General Hospital. All patients provided written informed consent for storage and use of their medical information in the hospital database for research purposes.

\section{IHC and pathologic evaluation}

IHC staining of ER, progesterone receptor (PR), HER2 and Ki-67 was routinely carried out by using Bench Mark GX system (Hoffman-La Roche Ltd., Basel, Switzerland) and was performed on $4 \mu \mathrm{m}$ slices of formalin-fixed,

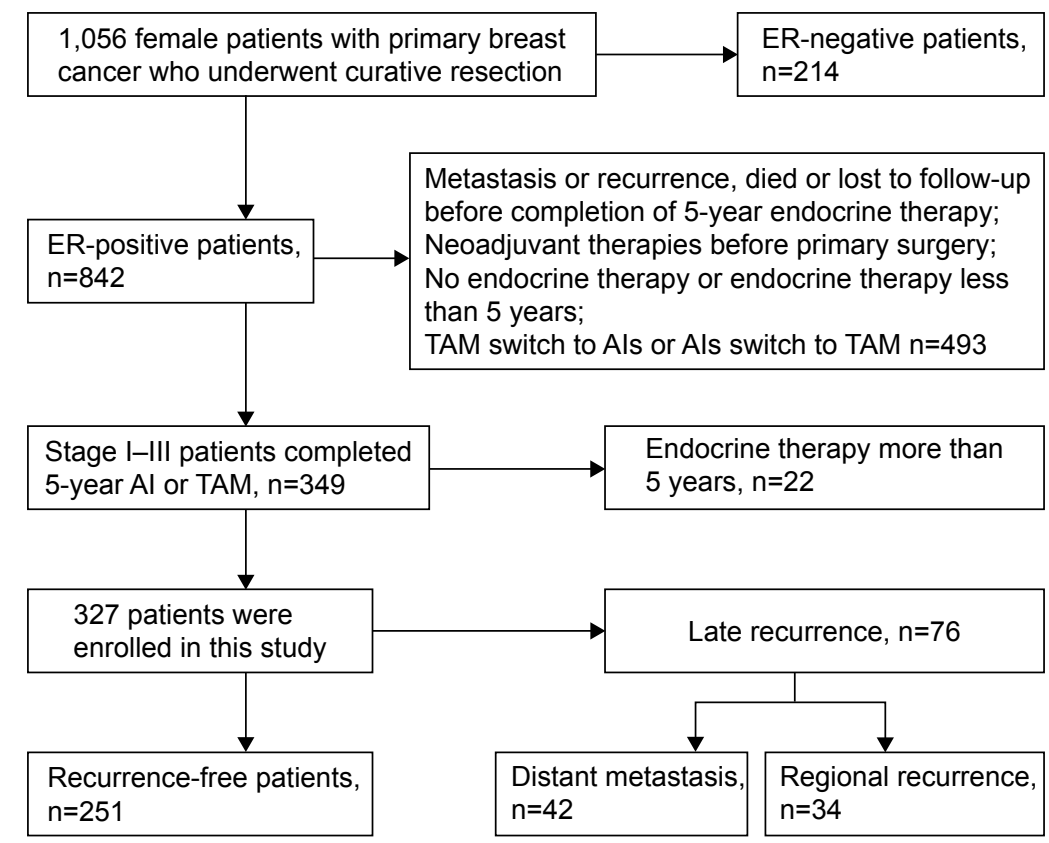

Figure I Flow diagram of patient recruitment.

Abbreviations: Als, aromatase inhibitors; ER, estrogen receptor; TAM, tamoxifen. 

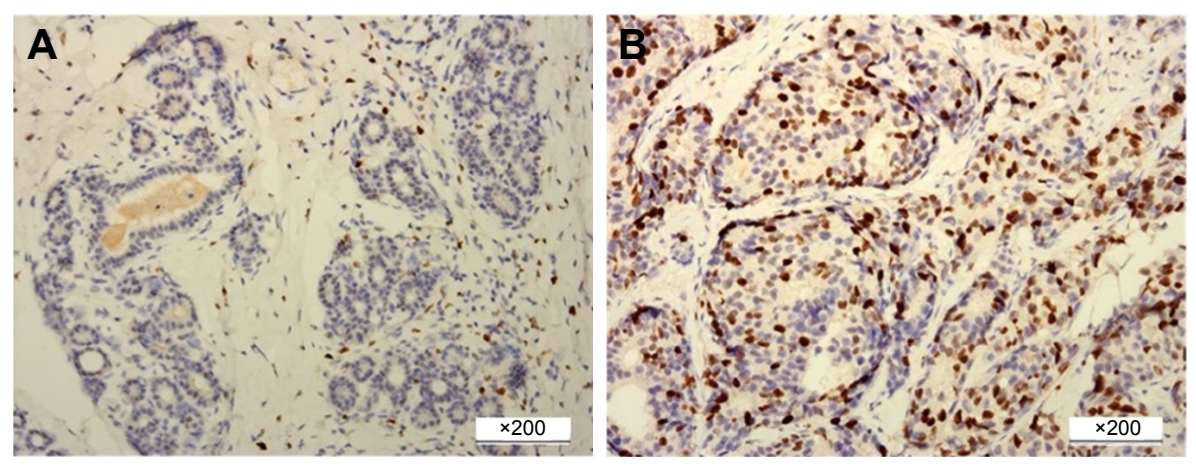

Figure 2 Immunohistochemical staining for $\mathrm{Ki}-67$ expression in breast cancer.

Notes: (A) Ki-67 low expression (<14\%), (B) Ki-67 high expression ( $\geq 14 \%)$. Original magnification $\times 200$.

paraffin-embedded tissue sections. The primary antibodies against ER (1:100 dilution), PR (1:150 dilution), HER2 (1:150 dilution) and Ki-67 (1:100 dilution) were purchased from Changdao Antibody Diagnostic Reagents Co. Ltd. (Shanghai, People's Republic of China). The cutoff for ER positivity and PR positivity was $1 \%$ positive tumor cells with nuclear staining. The Ki-67 index was expressed as the percentage of positively staining nuclear cells among at least 1,000 invasive cells in the area scored, with the staining intensity not being relevant. High Ki-67 index was defined as nuclear staining cells accounting for $14 \%$ or higher of all the diseased cells (Figure 2). ${ }^{15}$

Tissue sections were stained with hematoxylin and eosin. Histologic grade was evaluated according to the Nottingham grading system. ${ }^{16}$ Tumor grade consisted of three microscopic parameters: tubular formation, nuclear pleomorphism and mitotic counts. A score from 1 to 3 was assigned to each component, and the overall score was calculated to determine the histologic grade. A score of 3-5 points was recorded as grade 1, well differentiated; 5-6 points as grade 2, moderately differentiated; and 8-9 points as grade 3 , poorly differentiated. All histologic and IHC tumor slides were evaluated by experienced pathologists and examined by senior pathologists.

\section{Follow-up and survival end points}

The follow-up was primarily through telephone or data tracking of outpatient or hospital medical records. The contents of follow-up included survival situation, recurrence or no recurrence, adjuvant therapy, imaging examination and tumor marker levels. Latest follow-up was at the end of December 2016, with a median time of 8.5 years (5.6-10.9 years). The end point was recurrence-free survival (RFS) period, defined as the length of time from the date of diagnosis to any invasive breast cancer relapse (including locoregional or distant metastasis), or death, whichever occurred first or was censored at the date of last follow-up. Distant metastasis-free survival (DMFS) was also analyzed in this study.

\section{Statistical analysis}

Statistical analyses was performed using SPSS 20.0 software. Estimation of RFS and DMFS probability was performed using the Kaplan-Meier log-rank test. Univariate and multivariate analyses were performed using the Cox proportional hazards regression model to identify prognostic factors (including age, menopausal status, tumor size, pathologic type, histologic grade, node status, Ki-67, HER2, PR, TNM stage, endocrine therapy, chemotherapy and radiation therapy), and the variables were included in multivariate analysis when they were found to be statistically significant in univariate analysis. $P$-values $<0.05$ were considered significant.

\section{Results \\ Patients and characteristics}

A total of 327 patients with stage I-III invasive breast cancer were enrolled. All cases did not have distant metastasis and neoadjuvant therapy before surgery. The clinicopathologic characteristics of the study subjects are summarized in Table 1. A total of 141 (43.1\%) patients were $<50$ years, and $129(39.4 \%)$ patients were premenopausal. The tumors mainly consisted of invasive ductal carcinomas (82.6\%). Subdivisions based on the Ki-67 index were as follows: low proliferation 159 (48.6\%) and high proliferation 168 (51.4\%), with a cutoff of $14 \%$. PR was expressed in $84.4 \%$ of all cases, and $26.3 \%$ of the cases were HER2 positive. A total of 20 (6.1\%) patients died from breast cancer, 34 (10.4\%) patients developed a regional recurrence, and $42(12.8 \%)$ patients suffered from distant relapse. Treatment decision for every 
Table I Summary of patients' clinicopathologic characteristics and the associations with RFS and DMFS for all patients

\begin{tabular}{|c|c|c|c|c|c|}
\hline \multirow[t]{2}{*}{ Characteristics } & \multirow[t]{2}{*}{ No of patients (\%) } & \multicolumn{2}{|l|}{ RFS } & \multicolumn{2}{|l|}{ DMFS } \\
\hline & & Log-rank $\chi^{2}$ & $P$-value & Log-rank $\chi^{2}$ & $P$-value \\
\hline All patients & 327 & & & & \\
\hline Age (years) & & 0.019 & 0.892 & 0.096 & 0.757 \\
\hline$\leq 50$ & $|4|(43 . I)$ & & & & \\
\hline$>50$ & $186(56.9)$ & & & & \\
\hline Menopausal status & & 0.609 & 0.435 & 0.083 & 0.773 \\
\hline Premenopausal & $129(39.4)$ & & & & \\
\hline Postmenopausal & $198(60.6)$ & & & & \\
\hline Tumor size $(\mathrm{cm})$ & & 0.078 & 0.780 & 1.039 & 0.308 \\
\hline$\leq 2.0$ & 173 (52.9) & & & & \\
\hline$>2.0$ & $154(47.1)$ & & & & \\
\hline Number of LNM & & 11.819 & 0.003 & 12.995 & 0.002 \\
\hline 0 & $196(59.9)$ & & & & \\
\hline $1-3$ & $83(25.4)$ & & & & \\
\hline$\geq 4$ & $48(14.7)$ & & & & \\
\hline Pathologic type & & 1.179 & 0.278 & 0.731 & 0.393 \\
\hline IDC & $270(82.6)$ & & & & \\
\hline Non-IDC & $57(17.4)$ & & & & \\
\hline Histologic grade & & 16.290 & 0.000 & 12.539 & 0.000 \\
\hline $1 / 2$ & $24 \mid(73.7)$ & & & & \\
\hline 3 & $80(24.5)$ & & & & \\
\hline Unclear & $6(1.8)$ & & & & \\
\hline TNM stage & & 7.537 & 0.023 & 9.234 & 0.010 \\
\hline 1 & $122(37.3)$ & & & & \\
\hline II & $|5|(46.2)$ & & & & \\
\hline III & $54(16.5)$ & & & & \\
\hline HER2 & & 0.010 & 0.922 & 0.286 & 0.593 \\
\hline Negative or I+ & $24 I(73.7)$ & & & & \\
\hline $2+$ or $3+$ & $86(26.3)$ & & & & \\
\hline Chemotherapy & & 0.108 & 0.742 & 0.028 & 0.868 \\
\hline Yes & $285(87.2)$ & & & & \\
\hline No & $42(12.8)$ & & & & \\
\hline Radiation therapy & & 4.472 & 0.034 & 8.437 & 0.004 \\
\hline Yes & $105(32.1)$ & & & & \\
\hline No & $222(67.9)$ & & & & \\
\hline Endocrine therapy & & 0.397 & 0.529 & 1.002 & 0.317 \\
\hline TAM & $26 \mid(79.8)$ & & & & \\
\hline Als & $66(20.2)$ & & & & \\
\hline Ki-67 & & 7.134 & 0.008 & 4.594 & 0.032 \\
\hline High & I 68 (5I.4) & & & & \\
\hline Low & $159(48.6)$ & & & & \\
\hline PR & & 0.559 & 0.342 & 0.298 & 0.585 \\
\hline+ & $276(84.4)$ & & & & \\
\hline- & $5 I(I 5.6)$ & & & & \\
\hline
\end{tabular}

Notes: TNM stage is according to AJCC stage, 7th edition. Bold values show statistical significance $(P<0.05)$.

Abbreviations: Als, aromatase inhibitors; AJCC, American Joint Committee on Cancer; DMFS, distant metastasis-free survival; IDC, invasive ductal carcinoma; LNM, lymph node metastasis; PR, progesterone receptor; RFS, recurrence-free survival; TAM, tamoxifen.

patient was made according to the Chinese Anti-Cancer Association guidelines for the diagnosis and treatment of breast cancer. Also, 285 (87.2\%) patients underwent chemotherapy and 105 (32.1\%) patients underwent radiotherapy; $261(79.8 \%)$ patients received 5-year tamoxifen and 66 (20.2\%) patients received 5-year AIs (Table 1).

\section{Survival analysis}

A total of 76 patients (23.8\%) experienced distant metastasis or locoregional recurrence after completion of adjuvant endocrine therapy for 5 years. The main sites of metastasis were lung, bone, liver, local lymph nodes, contralateral breast, chest wall and brain. Figures 3 and 4 show that high histologic 

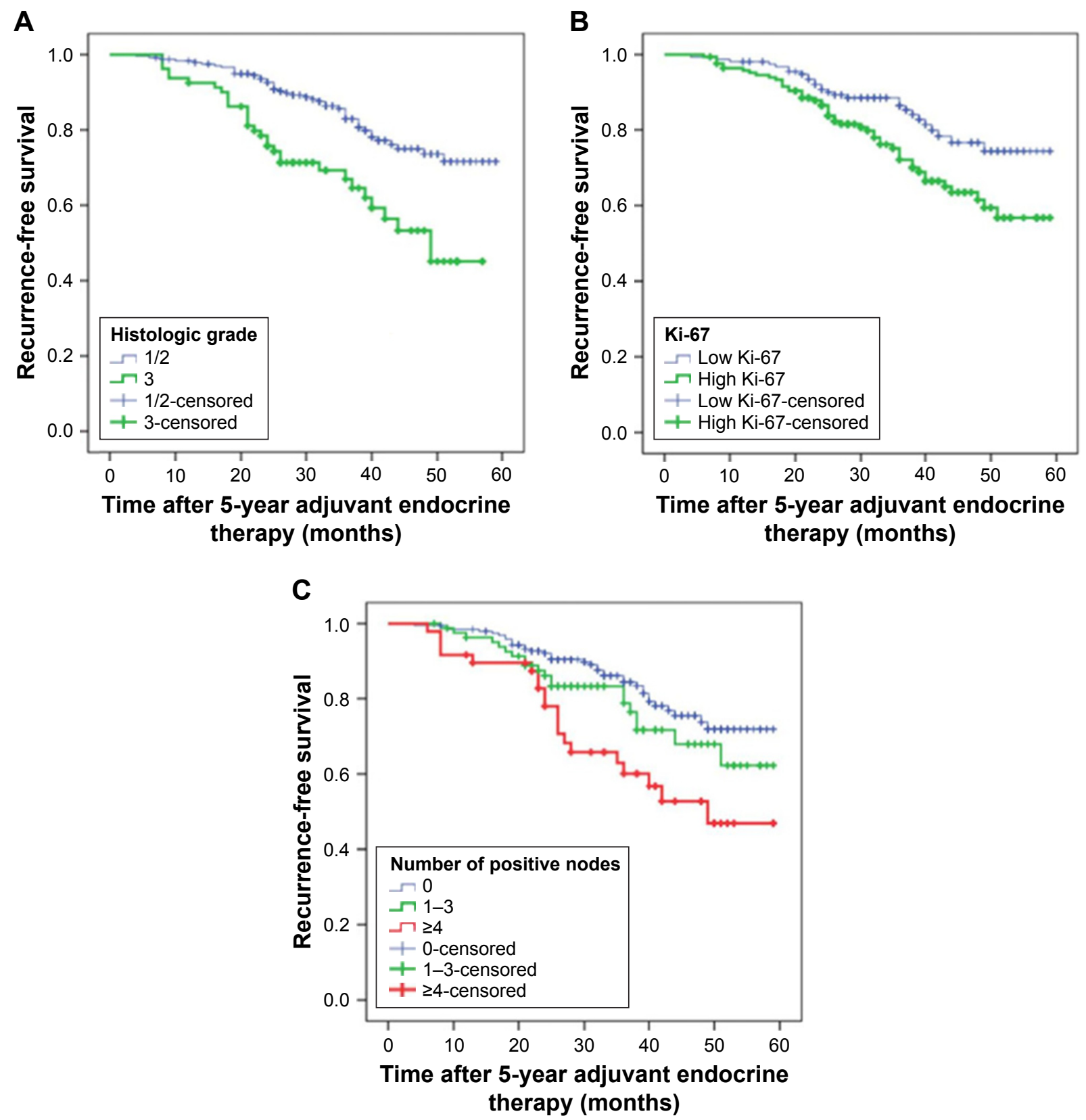

Figure 3 Kaplan-Meier plot of recurrence-free survival according to $(\mathbf{A})$ histologic grade, $P=0.000 ;(\mathbf{B}) \mathrm{Ki}-67, P=0.003$ and $(\mathbf{C})$ number of positive nodes, $P=0.008$.

grade, lymph node metastasis and high Ki-67 index were significantly associated with worse RFS ( $P=0.000,0.003$ and 0.008 , respectively; Figure 2; Table 1$)$ and DMFS ( $P=0.000$, 0.002 and 0.032 , respectively; Figure 3; Table 1).

\section{Risk factors for recurrence}

Risk factors that could potentially influence RFS were evaluated in univariate analyses for each of the 13 variables. Among these variables, five were found to be associated with a significant increase in the risk of locoregional recurrence and distant metastasis, including high histologic grade, lymph node metastasis, Ki-67 index, TNM stage and radiotherapy (Table 2). However, only two covariates were significantly associated with increased risk of locoregional recurrence and distant metastasis after adjustment for all other variables in the full multivariate model. Patients with more than three positive nodes had a hazard ratio (HR) of 2.176 (95\% CI=1.071-4.421, $P=0.032$ ) for recurrence, compared with patients with node-negative breast cancer. Patients with grade 3 tumors had an HR of 2.098 (95\% CI=1.300-3.385, $P=0.002)$ for recurrence, compared with patients with grade $1 / 2$ tumors (Table 3 ). 

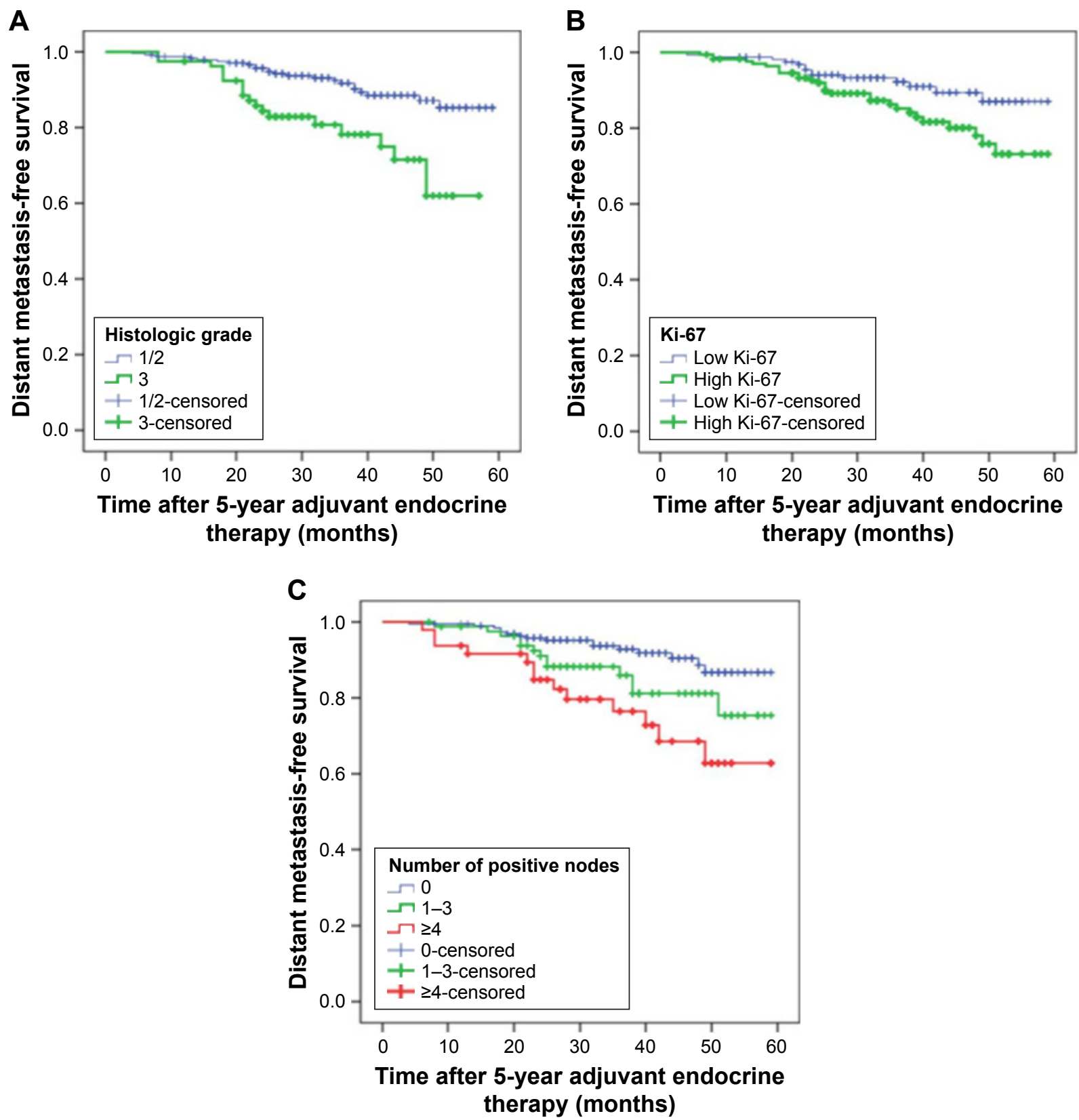

Figure 4 Kaplan-Meier plot of distant metastasis-free survival according to $(\mathbf{A})$ histologic grade, $P=0.000 ;(\mathbf{B}) \mathrm{Ki}-67, P=0.002$ and $(\mathbf{C})$ number of positive nodes, $P=0.032$.

\section{Risk factors for distant metastasis}

Risk factors that could potentially influence DMFS were evaluated in univariate analyses for each of the 13 variables. Among these variables, five were found to be associated with a significant increase in the risk of distant metastasis, including high histologic grade, lymph node metastasis, Ki-67 index, TNM stage and radiotherapy (Table 2). However, only histologic grade 3 (HR=2.212, 95\% CI $=1.166-4.194$; $P=0.015$ ) was significantly associated with increased risk of distant metastasis after elimination of all nonsignificant variables in the multivariate model (Table 3).

\section{Discussion}

Recently, Davies et al reported that continuing tamoxifen for 10 years rather than stopping at 5 years produced an absolute recurrence reduction of $3.7 \%$ and a mortality reduction of $2.8 \%$, most notably seen in the second decade after diagnosis. ${ }^{2}$ However, the extra benefit in terms of reduced recurrences and death shown in the study was modest, and therefore, it is appropriate to consider that not all patients with early breast cancer require extended endocrine therapy. Furthermore, potential side effects of additional endocrine therapy and noncompliance pose another issue in achieving 
Table 2 Univariate analysis for RFS and DMFS in ER+ breast cancer

\begin{tabular}{|c|c|c|c|c|c|c|}
\hline \multirow[t]{2}{*}{ Factors } & \multicolumn{3}{|l|}{ RFS } & \multicolumn{3}{|c|}{ DMFS } \\
\hline & HR & $95 \% \mathrm{Cl}$ of $\mathrm{HR}$ & $P$-value & HR & $95 \% \mathrm{Cl}$ of $\mathrm{HR}$ & $P$-value \\
\hline Radiation therapy & 1.624 & $1.030-2.562$ & 0.037 & 2.384 & $1.30|-4.37|$ & 0.005 \\
\hline Histologic grade & 2.480 & $1.568-3.922$ & 0.000 & 2.856 & $1.553-5.250$ & 0.001 \\
\hline $\mathrm{Ki}-67$ & 1.873 & I. $170-2.996$ & 0.009 & 1.987 & $1.046-3.775$ & 0.036 \\
\hline Node status & & & 0.004 & & & 0.003 \\
\hline I-3 vs 0 & 1.474 & $0.853-2.548$ & 0.164 & 2.088 & $1.004-4.343$ & 0.049 \\
\hline$>3$ vs 0 & 2.524 & $1.46 \mid-4.362$ & 0.001 & 3.535 & $|.700-7.35|$ & 0.001 \\
\hline TNM & & & 0.028 & & & 0.014 \\
\hline II vs I & 1.073 & $0.63 \mathrm{I}-1.826$ & 0.794 & 1.329 & $0.622-2.839$ & 0.463 \\
\hline III vs I & 2.062 & I.I54-3.683 & 0.015 & 2.999 & $1.36 \mathrm{I}-6.607$ & 0.006 \\
\hline Age & 1.032 & $0.654-1.627$ & 0.892 & 0.909 & $0.495-1.669$ & 0.757 \\
\hline Menopausal status & 1.203 & $0.754-1.918$ & 0.438 & 0.914 & $0.496-1.685$ & 0.773 \\
\hline Tumor size & 1.066 & $0.680-1.67 \mid$ & $0.78 \mathrm{I}$ & 1.369 & $0.746-2.514$ & 0.311 \\
\hline Pathologic type & 0.960 & $0.667-1.382$ & 0.825 & 0.667 & $0.350-1.270$ & 0.218 \\
\hline HER2 & 0.974 & $0.579-1.639$ & 0.922 & 1.201 & $0.6|3-2.35|$ & 0.594 \\
\hline Chemotherapy & 1.123 & $0.560-2.253$ & 0.743 & 1.083 & $0.425-2.755$ & 0.868 \\
\hline Endocrine therapy & 1.188 & $0.693-2.038$ & 0.531 & 0.646 & $0.272-1.533$ & 0.322 \\
\hline PR & 0.842 & $0.47 \mid-1.504$ & $0.56 \mathrm{I}$ & 0.807 & $0.374-1.745$ & 0.587 \\
\hline
\end{tabular}

Note: Bold values show statistical significance $(P<0.05)$.

Abbreviations: DMFS, distant metastasis-free survival; ER, estrogen receptor; HR, hazard ratio; PR, progesterone receptor; RFS, recurrence-free survival.

the best possible outcomes. ${ }^{3,7}$ Thus, it is critical to identify target populations with substantial risk of late recurrence to implement extended endocrine therapy, such that this therapy is cost-effective. ${ }^{4}$

The American Society of Clinical Oncology guideline recommended that women with hormone receptor-positive breast cancer who are pre- or perimenopausal should receive tamoxifen for an initial duration of 5 years, after which extended endocrine therapy is judged by menopausal status. ${ }^{3}$ The St Gallen consensus recommended that an extended endocrine therapy for a total of 10 years should be considered for premenopausal women with node-positive or with adverse pathology. ${ }^{17}$ This study newly found that patients with more than three positive nodes or grade 3 disease had substantial risk of recurrence, independent of endocrinotherapy regimens (tamoxifen or AIs), which is somewhat a complement of previous studies and suggests that patients with more than three positive nodes or grade 3 disease might require extended endocrine therapy. ${ }^{14,18,19}$

Pan Hongchao et al reported that even after 5 years of endocrine therapy, distant recurrence rates in years 5-20 were around $1 \%$ per year in $\mathrm{T} 1 \mathrm{~N} 0$ and much greater for those with T2N0 or node-positive disease. ${ }^{20}$ Our results showed that distant metastasis was still common (12.8\%) after 5-year adjuvant treatment and accounted for approximately half (55.3\%) of the recurrence, and that histologic grade 3 was the only independent variable that influenced distant metastasis after completion of 5-year adjuvant tamoxifen or AIs, which indicates that these patients might require extended therapy. Several reports showed similar results. ${ }^{18,19}$

The predictive role of biomarkers on extended treatment of breast cancer has been investigated in only a few

Table 3 Multivariable analyses of RFS and DMFS in ER+ breast cancer

\begin{tabular}{|c|c|c|c|c|c|c|}
\hline \multirow[t]{2}{*}{ Factors } & \multicolumn{3}{|l|}{ RFS } & \multicolumn{3}{|c|}{ DMFS } \\
\hline & HR & $95 \% \mathrm{Cl}$ of $\mathrm{HR}$ & $P$-value & HR & $95 \% \mathrm{Cl}$ of $\mathrm{HR}$ & $P$-value \\
\hline Radiation therapy & 1.064 & $0.589-1.922$ & 0.837 & I.49| & $0.697-3.192$ & 0.303 \\
\hline Histologic grade & 2.098 & $1.300-3.385$ & 0.002 & 2.212 & I.166-4.194 & 0.015 \\
\hline $\mathrm{Ki}-67$ & 1.477 & $0.902-2.417$ & 0.121 & 1.420 & $0.723-2.79 \mid$ & 0.309 \\
\hline Node status & & & 0.091 & & & 0.222 \\
\hline I-3 vs 0 & 1.170 & $0.659-2.078$ & 0.591 & 1.522 & $0.702-3.300$ & 0.287 \\
\hline$>3$ vs 0 & 2.176 & $|.07|-4.42 \mid$ & 0.032 & 2.245 & $0.894-5.637$ & 0.085 \\
\hline TNM & & & 0.290 & & & 0.494 \\
\hline II vs I & 0.839 & $0.485-1.450$ & 0.529 & 0.981 & $0.447-2.154$ & 0.963 \\
\hline III vs I & 1.379 & $0.693-2.745$ & 0.360 & 1.553 & $0.612-3.940$ & 0.355 \\
\hline
\end{tabular}

Note: Bold values show statistical significance $(P<0.05)$.

Abbreviations: DMFS, distant metastasis-free survival; ER, estrogen receptor; HR, hazard ratio; RFS, recurrence-free survival. 
studies with a focus on ER, PR, HER2 and Ki-67. A large size meta-analysis of early-stage breast cancer demonstrated that high Ki-67 was associated with a higher probability of relapse and worse survival. ${ }^{15,21}$ However, Ki-67 was not found to be an independent predictor of outcome, but high Ki-67 had a significantly higher risk of late recurrence in univariate analyses.

Whether the PR status affects the prognosis of ER-positive breast cancer patients is still controversial. Compared to ER-positive/PR-positive tumors, ER-positive/PR-negative tumors are frequently associated with upregulation of specific oncogenic pathways, including PI3K/Akt/mTOR. ${ }^{13,22}$ However, in this study, PR status was not significantly associated with late RFS or DMFS, which was similar to the results of the BIG 1-98 trial and the ATAC trial. ${ }^{8,9}$ Beyond this, we found that HER2 was also not associated with late recurrence in ER-positive patients, which might be attributed to the small size of patients with a high expression of HER2 $(26.3 \%)$ and was similar to the results of the ATAC trial and different with the BIG 1-98 trial. ${ }^{8,9}$

In addition to specific clinical and pathologic features, multigene expression signatures are capable of predicting the risk of late recurrence after treatment in patients with ER-positive breast cancer. ${ }^{18}$ Currently, only three multiparameter gene expression tests, the PAM50 risk of recurrence, Breast Cancer Index and the Endopredict assay, are shown to be prognostic beyond 5 years and may potentially discriminate patients into low and high risk for late distant recurrence. ${ }^{23-25}$ However, currently, clinicians should not make decisions based on such assays because there is no consensus about how low the annual risk of recurrence should be to avoid extended adjuvant endocrine therapy. 8,26

Nonetheless, extended endocrine therapy is a promising strategy to further reduce the risk of recurrence. ${ }^{4,14}$ Prospective studies are still needed to confirm the results given above, and emphasis should be laid on refining the evidence to include subpopulations of patients who would benefit most from extended endocrine therapy. By tailoring for individual treatment, overtreatment, side effects and unnecessary costs will be prevented and great value will be gained in the treatment of breast cancer.

\section{Conclusion}

Late relapse after completion of 5-year adjuvant endocrine therapy was still common, and grade 3 and more than three positive nodes were the risk factors of late recurrence; however, grade 3 was the only risk factor of late distant metastasis. Our results might be helpful to identify patients with higher risk, who may be candidates for extended endocrine therapy. With the available data, the decision making as regards to extended endocrine treatment should, therefore, focus not only on the cancer biology alone, but also include assessment of individual risk of late recurrence based on patient's clinicopathologic characteristics and available molecular signatures, menopausal status, treatment side effects and patients' preference.

\section{Acknowledgments}

This work is supported by the scientific research project of Shanghai Science and Technology Committee (No 14411950205, 14411950200).

\section{Disclosure}

The authors report no conflicts of interest in this work.

\section{References}

1. Early Breast Cancer Trialists' Collaborative Group (EBCTCG). Relevance of breast cancer hormone receptors and other factors to the efficacy of adjuvant tamoxifen, patient-level meta-analysis of randomised trials. Lancet. 2011;378(9793):771-784.

2. Davies C, Pan H, Godwin J, et al; Adjuvant Tamoxifen: Longer Against Shorter (ATLAS) Collaborative Group. Long-term effects of continuing adjuvant tamoxifen to 10 years versus stopping at 5 years after diagnosis of oestrogen receptor-positivebreast cancer: ATLAS, a randomised trial. Lancet. 2013;381(9869):805-816.

3. Burstein HJ, Temin S, Anderson H, et al. Adjuvant endocrine therapy for women with hormone receptor-positive breast cancer: American society of clinical oncology clinical practice guideline focused update. J Clin Oncol. 2014;32(21):2255-2269.

4. Goss PE, Ingle JN, Pritchard KI, et al. Extending aromatase-inhibitor adjuvant therapy to 10 years. $N$ Engl J Med. 2016;375(3):209-219.

5. Blows FM, Driver KE, Schmidt MK, et al. Subtyping of breast cancer by immunohistochemistry to investigate a relationship between subtype and short and long term survival: a collaborative analysis of data for 10,159 cases from 12 studies. PLoS Med. 2010;7(5):e1000279.

6. Fisher B, Dignam J, Bryant J, Wolmark N. Five versus more than five years of tamoxifen for lymph node-negative breast cancer: updated findings from the National Surgical Adjuvant Breast and Bowel Project B-14 randomized trial. J Natl Cancer Inst. 2001;93(9):684-690.

7. Howell A, Cuzick J, Baum M, et al; ATAC Trialists' Group. Results of the ATAC (Arimidex, Tamoxifen, Alone or in Combination) trial after completion of 5 years' adjuvant treatment for breast cancer. Lancet. 2005;365(9453):60-72.

8. Mauriac L, Keshaviah A, Debled M, et al; BIG 1-98 Collaborative Group; International Breast Cancer Study Group. Predictors of early relapse in postmenopausal women with hormone receptor-positive breast cancer in the BIG 1-98 trial. Ann Oncol. 2007;18(5):859-867.

9. Dowsett M, Allred C, Knox J, et al. Relationship between quantitative estrogen and progesterone receptor expression and human epidermal growth factor receptor 2 (HER-2) status with recurrence in the Arimidex, Tamoxifen, Alone or in Combination trial. J Clin Oncol. 2008;26(7): 1059-1065.

10. Duggan C, Marriott K, Edwards R, Cuzick J. Inherited and acquired risk factors for venous thromboembolic disease among women taking tamoxifen to prevent breast cancer. J Clin Oncol. 2003;21(19):3588-3593.

11. Fornander T, Rutgvist LE, Cedermark B, et al. Adjuvant tamoxifen in early breast cancer: occurrence of new primary cancers. Lancet. 1989;1(8630):117-120. 
12. Baum M, Budzar AU, Cuzick J, et al; ATAC Trialists' Group. Anastrozole alone or in combination with tamoxifen versus tamoxifen alone for adjuvant treatment of postmenopausal women with early breast cancer: first results of the ATAC randomised trial. Lancet. 2002; 359(9324):2131-2139.

13. Koboldt DC, Fulton RS, McLellan MD, et al; Cancer Genome Atlas Network. Comprehensive molecular portraits of human breast tumours. Nature. 2012;490(7418):61-70.

14. Li J, Jiang Y, Liu Y, Shao Z. Identify high risk estrogen receptorpositive breast cancer patients for extended endocrine therapy. Breast. 2017:31:173-180.

15. Cheang MC, Chia SK, Voduc D, et al. Ki67 index, HER2 status, and prognosis of patients with luminal B breast cancer. J Natl Cancer Inst. 2009;101(10):736-750.

16. Elston CW, Ellis IO. Pathological prognostic factors in breast cancer. The value of histological grade in breast cancer: experience from a large study with long-term follow-up. Histopathology. 1991;19(5): 403-410.

17. Coates AS, Winer EP, Goldhirsch A, et al; Panel Members. Tailoring therapies-improving the management of early breast cancer: St Gallen international expert consensus on the primary therapy of early breast cancer 2015. Ann Oncol. 2015;26(8):1533-1546.

18. Sestak I, Dowsett M, Zabaglo L, et al. Factors predicting late recurrence for estrogen receptor-positive breast cancer. J Natl Cancer Inst 2013;105(19):1504-1511.

19. Kennecke HF, Olivotto IA, Speers C, et al. Late risk of relapse and mortality among postmenopausal women with estrogen responsive early breast cancer after 5 years of tamoxifen. Ann Oncol. 2007;18(1) 45-51.
20. Pan Hongchao, Gray Richard G, Davies Christina, et al. For the Early Breast Cancer Trialists' Collaborative Group (EBCTCG). Predictors of recurrence during years 5-14 in 46,138 women with ER + breast cancer allocated 5 years only of endocrine therapy (ET). Poster presented at: Annual meeting ASCO; 2016. J Clin Oncol. 34 (suppl; abstr 505).

21. de Azambuja E, Cardoso F, de Castro G Jr, et al. Ki-67 as prognostic marker in early breast cancer: a meta-analysis of published studies involving 12,155 patients. Br J Cancer. 2007;96(10):1504-1513.

22. Creighton CJ, Kent Osborne C, van de Vijver MJ, et al. Molecular profiles of progesterone receptor loss in human breast tumors. Breast Cancer Res Treat. 2009;114(2):287-299.

23. Jinih M, Relihan N, Corrigan MA, O'Reilly S, Redmond HP. Extended adjuvant endocrine therapy in breast cancer: evidence and update a review. Breast J. Epub 2017 Mar 2.

24. Sgroi DC, Sestak I, Cuzick J, et al. Prediction of late distant recurrence in patients with oestrogen-receptor-positive breast cancer: a prospective comparison of the breast-cancer index (BCI) assay, 21-gene recurrence score, and IHC4 in the TransATAC study population. Lancet Oncol. 2013;14(11):1067-1076.

25. Zhang Y, Schnabel CA, Schroeder BE, et al. Breast cancer index identifies early-stage estrogen receptor-positive breast cancer patients at risk for early- and late-distant recurrence. Clin Cancer Res. 2013;19(15): 4196-4205.

26. Harris LN, Ismaila N, McShane LM, et al. Use of biomarkers to guide decisions on adjuvant systemic therapy for women with early-stage invasive breast cancer: American society of clinical oncology clinical practice guideline. J Clin Oncol. 2016;34(10):1134-1150.
OncoTargets and Therapy

\section{Publish your work in this journal}

OncoTargets and Therapy is an international, peer-reviewed, open access journal focusing on the pathological basis of all cancers, potential targets for therapy and treatment protocols employed to improve the management of cancer patients. The journal also focuses on the impact of management programs and new therapeutic agents and protocols on

\section{Dovepress}

patient perspectives such as quality of life, adherence and satisfaction. The manuscript management system is completely online and includes a very quick and fair peer-review system, which is all easy to use. Visit http://www.dovepress.com/testimonials.php to read real quotes from published authors. 